Research Paper

\title{
Effects of Hypoxia, Surrounding Fibroblasts, and p16 Expression on Breast Cancer Cell Migration and Invasion
}

\author{
Jun Zhang, Liyuan Li and Yi Lu \\ Department of Pathology and Laboratory Medicine, University of Tennessee Center for Cancer Research, University of Tennessee Health \\ Science Center, Memphis, Tennessee, USA.
}

\begin{abstract}
$\square$ Corresponding author: Yi Lu, Ph.D. Department of Pathology and Laboratory Medicine, University of Tennessee Health Science Center, Cancer Research Building, Room 218, 19 South Manassas Street, Memphis, TN 38163, USA. Telephone: (901) 448-5436 Fax: (901) $448-5496$ e-mail: ylu@uthsc.edu.
\end{abstract}

๑) 2015 Ivyspring International Publisher. Reproduction is permitted for personal, noncommercial use, provided that the article is in whole, unmodified, and properly cited. See http://ivyspring.com/terms for terms and conditions.

Received: 2014.12.15; Accepted: 2015.01.05; Published: 2015.03.06

\begin{abstract}
Cancer cell migration and invasion play essential roles in the metastatic cascade that transforms the local, noninvasive confined tumor cells to the motile, metastatic cancer cells moving through the extracellular matrix and basement into the circulation. Accumulated evidences suggest that intratumoral hypoxia, a characteristic of fast-growing solid tumors, promotes cancer cell motile and invasive abilities. In this study, we investigated the effects of hypoxia, surrounding fibroblasts, and p16 expression on the migration and invasion of breast cancer cells. We found that hypoxia promoted breast cancer cell migration and invasion, and cocultured fibroblasts stimulated invasiveness of breast cancer cells. Moreover, by using a Tet-on inducible system, we found that p16 is capable of inhibiting hypoxia-induced cell migration and invasion of breast cancer cells, and suppressing cocultured fibroblast-stimulated invasiveness of breast cancer cells. These results suggest that p16, in addition to its well-known anti-tumor proliferation function, has novel anti-cancer properties capable of suppressing hypoxia-mediated cancer cell migration and invasion. This study may provide important validation for p16-mediated cancer therapy either by gene therapy or pharmacological activation of internal p16 gene that is usually inactive due to hypermethylation in the tumor cells.
\end{abstract}

Key words: breast cancer, fibroblasts, hypoxia, invasion, migration, p16.

\section{Introduction}

One key characteristic of fast-growing solid tumors is the development of intratumoral hypoxia, which promotes malignant tumor progression. Cancer cell migration and invasion play important roles in the metastatic cascade. Cell migration is the transition process from the local, noninvasive confined tumor cells to the migrating, metastatic cancer cells when the cells obtain the ability to dissociate from intracellular adhesions and become motile $(1,2)$. Invasion, on the other hand, is the process by which malignant cells move through the basement membrane and gain ac- cess to blood vessels and lymphatic channels. As cancer cell migration and invasion are essential prerequisites for tumor metastasis, identifying agents that effectively block hypoxia-induced cancer migration and invasion would promote development of effective anti-cancer therapeutic approaches.

Hypoxia-inducible factor-1 (HIF-1), a master transcriptional factor in response to hypoxia, activates a group of downstream genes that promote tumor malignant progression. HIF-1 is composed of an inducible subunit, HIF- $1 \alpha$ and a constitutively ex- 
pressed subunit, HIF-1ß (3). Heterodimerization of HIF- $1 \alpha$ and HIF- $1 ß$ is required to form the transcriptionally active HIF-1 that binds to the hypoxic response elements within the promoter regions of target genes (4). HIF-1 $\alpha$ degrades quickly under normal oxygen tension (5) but it is inducible and stabilized under low oxygen tension (hypoxia) (6). Increased HIF-1 $\alpha$ levels have been shown to correlate with decreased patient survival in many cancers including breast cancer (BCa) (7). The activation of HIF-1 $\alpha$ stimulates a group of HIF-1 $\alpha$-regulated genes including vascular endothelial growth factor (VEGF) $(3,8)$. As a result, the cells are converted towards malignant progression. HIF-1 activity is mainly dependent on the level of HIF- $1 \alpha$ protein, the inducible and regulatory subunit of the HIF-1 dimer complex (9, 10).

We have previously shown that p16, a tumor suppressor gene and cyclin D kinase inhibitor and a negative cell cycle regulator (11), can neutralize the transactivation ability of HIF-1 $\alpha$ on its target gene VEGF (12). In this study, we evaluated whether hypoxia stimulates breast cancer migration and invasion, and whether ectopic expression of p16 is capable of modulating hypoxia-mediated cell migration and invasion. We also evaluated whether cocultured stromal cells (fibroblasts) promotes BCa cell invasion and whether p16 inhibits that effect, as well as whether HIF-1a of fibroblasts specifically has a role in promoting cocultured BCa cell invasiveness.

\section{Materials and Methods}

\section{Cell lines, cell culture conditions, and reagents}

Human breast cancer (BCa) cell line MDA-MB-231 was obtained from American Type Culture Collection (ATCC, Manassas, VA). Human breast cancer cell lines LM2, a MDA-MB-231 derivative line that has high lung metastatic ability (15), was a generous gift from Dr. J. Massague of Memorial Sloan-Kettering Cancer Center (NY). Murine mammary carcinoma cell line JygMC(A) was from Dr. H. Azuma of Osaka Medical College, Osaka, Japan (13). Mouse MEF fibroblasts expressing wild-type (WT) and knockout (KO) HIF-1 $\alpha$ were from Dr. T Seagroves of University of Tennessee Health Science Center (TN).

MDA-MB-231 cells were grown in RPM1-1640 (Gibco, Life technologies Corporation, Grand Island, NY) with $10 \%$ fetal bovine serum (FBS) (Gibco), LM2 cells were grown in Dulbecco's Modified Eagle medium (DMEM) (Cellgro, Mediatech, Inc, Manassas, VA) with $10 \%$ FBS. JygMC(A) cells were grown in DMEM (Cellgro) with 10\% FBS. Both MEF lines were grown in DMEM medium with 25 mM HEPES (Gib- co) with $10 \%$ FBS. All cell lines were grown in medium containing 100 units $/ \mathrm{ml}$ penicillin, and 100 $\mu \mathrm{g} / \mathrm{ml}$ streptomycin. The cell cultures were incubated at $37^{\circ} \mathrm{C}$ either under normoxia $\left(5 \% \mathrm{CO}_{2}, 21 \% \mathrm{O}_{2}\right)$ or hypoxia $\left(5 \% \mathrm{CO}_{2}, 1 \% \mathrm{O}_{2}\right.$, balanced with $\left.\mathrm{N}_{2}\right)$ conditions.

\section{Generation and screen of stably transfected, Dox inducible (Tet-on) p16-expressing breast cancer cell lines}

The construction of the Tet-on p16 lentiviral system Lenti-Tet-on p16 (pLenti-Tet-p16-pgkpuro and pcFUW-rtTA3-IRES-puro) was described previously (12). Breast cancer cells (MDA-MB-231, LM2, and JygMC(A), respectively) were co-transduced by pLenti-Tet-p16-pgkpuro and pcFUW-rtTA3-IRESpuro at multiplicity of infection (moi) of 10 each, together with $6 \mu \mathrm{g} / \mathrm{ml}$ polybrene (Millipore, Bedford, MA). The stably transfected cells were enriched in medium containing $1 \mu \mathrm{g} / \mathrm{ml}$ puromycin (Clontech, Mountain View, CA). The resultant stably transfected cells are named as MDA/Tet-on-p16, JygMC(A)/Tet-on-p16, and LM2/Tet-on-p16, respectively. For induction of p16 transgene expression in Tet-on-p16 stably transfected cells, $1 \mu \mathrm{g} / \mathrm{ml}$ doxycyline (Dox) (Clontech) was used in the medium for incubation.

\section{Immunohistochemistry}

The procedure followed the method as described previously (12). Briefly, for immunohistochemical (IHC) staining, cultured cells were grown on SlideFlasks with bottom detachable slides (Nalge Nunc, Naperville, IL) that could be used for IHC staining directly later. The samples (slides) were first incubated with $1 \% \mathrm{H}_{2} \mathrm{O}_{2}$ for $30 \mathrm{~min}$. The samples were incubated with first antibody against human p16 (Santa Cruz Biotechnology, Inc., Santa Cruz, CA) for $16 \mathrm{~h}$ at $4^{\circ} \mathrm{C}$, then by corresponding second antibody and the Universal Elite ABC Kit (Vector Laboratories, Inc., Burlingame, $\mathrm{CA}$ ) according to the manufacturer's protocol. The reaction was visualized with DAB solution (75 mg 3,3'-Diaminobenzidine and $30 \mathrm{ml} 50 \%$ $\mathrm{H}_{2} \mathrm{O}_{2}$ in $150 \mathrm{ml} \mathrm{PBS}$ ) for 3-10 min.

\section{Cell migration assay}

The breast cancer MDA-MB-231 cells stably transfected with inducible Tet-on p16 (MDA/Tet-on p16) were incubated in the absence or presence of 1 $\mu \mathrm{g} / \mathrm{ml}$ Dox for $72 \mathrm{~h}$. The cells were then harvested and used for the following migration assay. The cell migration was measured by a modified Boyden's chamber method using BD Falcon Cell Culture Inserts incorporating polyethylene terephthalate (PET) track-etched membrane with a pore size of $8.0 \mu \mathrm{m}$ (BD 
Bioscience, Belgium). The inserts were precoated on the under-surface (between upper and lower chambers) with $10 \mu \mathrm{g} / \mathrm{ml}$ fibronectin (Thermo Fisher Scientific) at $37^{\circ} \mathrm{C}$ for $3 \mathrm{~h}$. Above mentioned harvested cells in suspension of serum-free medium (SFM) with $1 \%$ BSA were incubated at $37^{\circ} \mathrm{C}$ for $30 \mathrm{~min}$ under normoxic $\left(5 \% \mathrm{CO}_{2}, 21 \% \mathrm{O}_{2}\right)$ or hypoxic $\left(5 \% \mathrm{CO}_{2}, 1 \%\right.$ $\mathrm{O}_{2}$, balanced with $\mathrm{N}_{2}$ ) conditions. Subsequently, cell suspensions were seeded into the upper chamber of an insert at a density of 10,000 cells per well, and 300 $\mu \mathrm{l}$ serum-free medium with $1 \%$ BSA was placed in the lower chamber to act as a chemoattractant in the 24-well cell culture plate. The cells were further incubated at normoxia and hypoxia (see above) at $37^{\circ} \mathrm{C}$ for $3 \mathrm{~h}$. The inserts were then removed and nonmigrating cells remaining on the upper side of the filter were scraped off. The cells that had migrated to the lower surface of the insert were stained using Giemsa staining solution (Sigma, St. Louis, MO). After extensive washing with water, the migrated cells were counted in five different fields under a microscope at x200 magnification. Migratory activity was expressed as the number of cells (that is, the sum of total cell numbers in five randomly selected fields of view) that migrated to the lower side of the filter.

\section{In vitro invasion assay with commercially pre- coated invasion inserts}

The BCa cells were examined by the invasion assay under normoxia by using commercially available invasion inserts. For each Dox inducible BCa/Tet-on p16 cell line, the cells were either incubated in medium with or without $1 \mu \mathrm{g} / \mathrm{ml}$ Dox for 72 $h$. The cells were then harvested and used for the following in vitro invasion assay in the 6-well-plate $\mathrm{BD}$ Biocoat Matrigel Invasion Chambers (BD Biosciences Bio-Coat Matrigel Invasion Chamber) according to the manufacturer's procedure. Briefly, the chamber was first rehydrated with serum-free medium (SFM) for 2 $\mathrm{hr}$ at $37^{\circ} \mathrm{C}$. After rehydration, the chambers were placed in the lower compartment loaded with medium containing 5\% FBS. Meanwhile, the above-mentioned cells were suspended and adjusted to $1.25 \times 10^{5}$ cells $/ \mathrm{ml}$ in SFM with or without $1 \mu \mathrm{g} / \mathrm{ml}$ Dox. The cell suspension $\left(2 \mathrm{ml}\right.$ or $2.5 \times 10^{5}$ cells per well) was immediately added to the upper compartment of the chamber. The cells were then allowed to invade through the matrigel for $22 \mathrm{~h}$ at $37^{\circ} \mathrm{C}$, and the noninvading cells were removed by scrubbing the upper surface with a wet cotton swab. The filters were stained with Diff-Quick stain kit (Dade Behring Inc., Newark, DE), drained and counted under the microscope.

\section{In vitro invasion assay with self-coated matrigel}

The BCa LM2 cells, which express a green fluorescent protein (GFP) gene (15), were transfected with Lenti-Tet-on p16 to generate LM2/Tet-on-p16 line. LM2/Tet-on-p16 cells were incubated in the absence or presence of $1 \mu \mathrm{g} / \mathrm{ml}$ Dox for $72 \mathrm{~h}$ before the experiment, and the samples with Dox induction were maintained throughout the following assay. All the cells (for simplicity, we used LM2 here referring LM2 or LM2/Tet-on-p16, and MEF referring MEF/HIF-1 $\alpha$ WT or MEF/HIF-1 $\alpha \mathrm{KO}$ ) were grown in SFM overnight and then harvested for the following invasion assay. The 24-well size Falcon Cell Culture Inserts (Corning Incorporated, Corning, NY) were precoated on the upper-surface (inside the inserts) with $100 \mu \mathrm{l}$ matrigel (BD Matrigel, Bedford, MA) per insert and let them solidify at $37^{\circ} \mathrm{C}$ overnight. For experiment with BCa cells alone, cell suspension of $5 \times 10^{5}$ LM2 cells in $100 \mu \mathrm{l}$ SFM was seeded into the upper chamber of the insert; for experiment with coculture of $\mathrm{BCa}$ and fibroblasts, cell suspension of $5 \times 10^{5} \mathrm{LM} 2$ cells and $5 \times 10^{5}$ MEF cells in $100 \mu \mathrm{l}$ SFM was seeded into the upper chamber of the insert. For the lower chamber of a 24-well cell culture plate, $650 \mu \mathrm{l}$ SFM with $5 \%$ BSA (alternatively, with 5\% FBS) was placed. The cells were then incubated at either normoxic or hypoxic conditions (see above) at $37^{\circ} \mathrm{C}$ for $18 \mathrm{~h}$. The inserts were then removed, the matrigel (containing the unpenetrated cells) in the upper chamber of the inserts was carefully wiped off by using a wet cotton-tipped applicator. The resultant inserts were placed on a clean 24-well plate, and the matrigel-penetrated/invaded $\mathrm{BCa}$ LM2 cells on the membrane of the inserts were measured by using the SpectraMax M2 microplate reader (Molecular Devices, Sunnyvale, CA) for counting the GFP fluorescence intensity (RFU). Because LM2 line expresses GFP, this approach would only count the invaded BCa LM2 (GFP-expressing) cells.

\section{Results}

\section{Hypoxia stimulates BCa cell migration and p16 inhibits this hypoxia-induced migration}

Cell migration is an important aspect of the tumor metastatic process. HIF-1a was implied, by others' studies, in stimulation of cancer cell migration $(16,17)$ and hypoxia induces HIF-1a $(14)$. Thus, we were interested in analyzing whether hypoxia has effect on breast cancer cell migration and whether p16, a biological agent that appears to neutralize HIF-1a transcriptional activity (12), has ability to suppress hypoxia-mediated cancer cell migration.

To accurately examine the effects of p16 on BCa cell migration, we designed an inducible p16 expres- 
sion so that $\mathrm{p} 16$ expression and effects can be studied in the same cells with or without inducer. A lentivirus expressing inducible human wild-type (WT) p16 under the control of a Tet-on promoter (Lenti-Tet-on p16) was constructed and stably transfected into MDA-MB-231 cells. The resultant stably infected line (MDA/Tet-on-p16) demonstrated a tightly regulated and inducible p16 expression under inducer Dox treatment (+Dox, top panel) (Fig. 1). Importantly, MDA-MB-231 cells, like other BCa cell lines (including JygMC(A) and LM2) we have examined, do not express endogenous p16 protein in the absence of Dox (-Dox) induction (Fig. 1, top panel). In other words, MDA/Tet-on-p16 cells in the absence of Dox behave just as control parental MDA-MB-231 cells.

MDA/Tet-on-p16 line was subsequently used to detect potential effects that $\mathrm{p} 16$ had on cell migration. A modified Boyden's chamber assay using transwell precoated with fibronectin (a component of extracellular matrix) onto the insert undersurface of the membrane was used as described in the $M$ \& $M$ for the migration measurement. As shown in Fig. 1 (bottom panel), MDA/Tet-on p16 cells under hypoxia had more than two-fold (>100\%) increase in cell migration than that of normoxia (compare first two columns on the left). Importantly, inducible p16 expression by
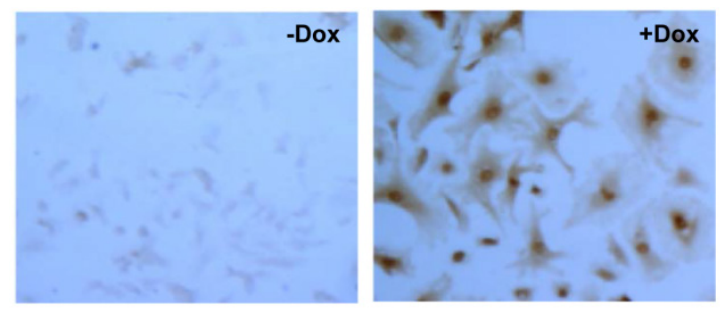

MDA/Tet-on-p16

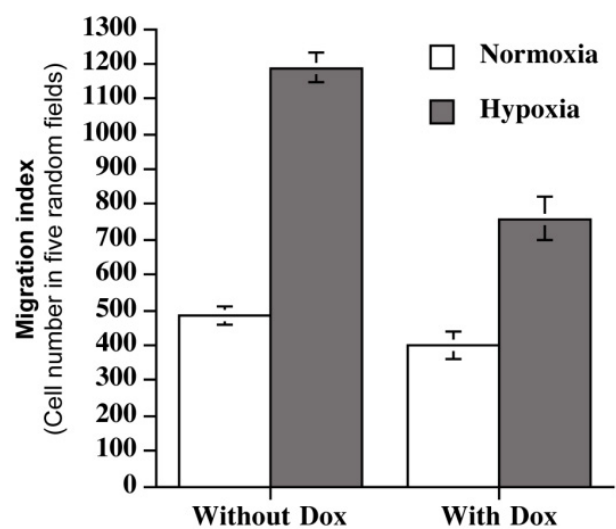

Figure 1. Inducible p16 expression in MDA-MB-231 cells and p16's effect on hypoxia-induced migration. (Top panel) IHC staining for p16 protein in MDA-MB-231 cells stably expressing Tet-on inducible p16 (MDA/Tet-on-p16): MDA/Tet-on-p16 cells were treated without or with $1 \mu \mathrm{g} / \mathrm{ml}$ Dox for $72 \mathrm{~h}$. The cells were then IHC stained by primary anti-p16 antibody. The dark brown color indicates pl6 protein (+Dox). (Bottom panel) pl6 inhibits hypoxia-induced cell migration: MDA/Tet-on-p16 cells were incubated with or without $1 \mu \mathrm{g} / \mathrm{ml}$ Dox for $72 \mathrm{~h}$, followed by cell migration assay as described in $M$ \& $M$ section under normoxic and hypoxic conditions. The results represent the data from at least two independent experiments, each performed in triplicate.
Dox significantly inhibited this hypoxia-induced cell migration (column 4) with a $36.0 \%$ inhibition in comparison to cells under hypoxia without p16 expression (column 2), indicating that p16 effectively inhibits hypoxia-induced migration in MDA-MB-231 cells. In contrast, p16's effect on cell migration under normoxia is marginal. It should be noted that the inhibition of migration is not due to p16-mediated effect on cell proliferation, as the migration assay was conducted within $3 \mathrm{~h}$ and the MDA-MB-231 cell line's doubling time is $41.9 \mathrm{~h}$. In addition, Dox treatment per se did not have any effect on migration as evidenced by Dox treatment on BCa cells stably transfected with inducible GFP (not shown). These combined results (Fig. 1) strongly demonstrate that hypoxia stimulates BCa cell migration and p16 significantly inhibits this hypoxia-induced migration.

\section{p16 inhibits invasiveness ability of BCa cells}

Invasion is the process by which malignant cells move through the basement membrane and gain access to blood vessels and lymphatic channels. The classic and well-established in vitro invasion assay aims to measure cancer cells' ability to penetrate the matrigel (a resemble mixture of basement membrane matrix).

p16's effect on cancer cell invasiveness has never been studied before. To investigate the effects of p16 on BCa invasive ability, we used the in vitro invasion assay to analyze three BCa lines' abilities to penetrate matrigel, in the presence or absence of p16 expression, respectively. Briefly, BCa cells stably transfected with inducible Tet-on p16 were incubated with or without 1 $\mu \mathrm{g} / \mathrm{ml}$ Dox for 3 days, and then the treated $\mathrm{BCa}$ cells were placed on the matrigel invasion chamber (BD Biosciences Bio-Coat Matrigel Invasion Chamber). After 22 hours incubation at $37^{\circ} \mathrm{C}$, the noninvasive cells were carefully removed from the upper surface of the membrane, and the penetrated cells (i.e., the invasive cells) going through matrigel were counted under the microscope. We found that all three BCa cell lines had significantly reduced invasiveness in the presence of p16 (Dox induced), at a decrease of $85.8 \%$ (MDA-MB-231), 82.7\% (JygMC(A)), and $80.4 \%$ (LM2), compared to their corresponding untreated (p16 non-expressing) counterparts, respectively (Fig. 2). 


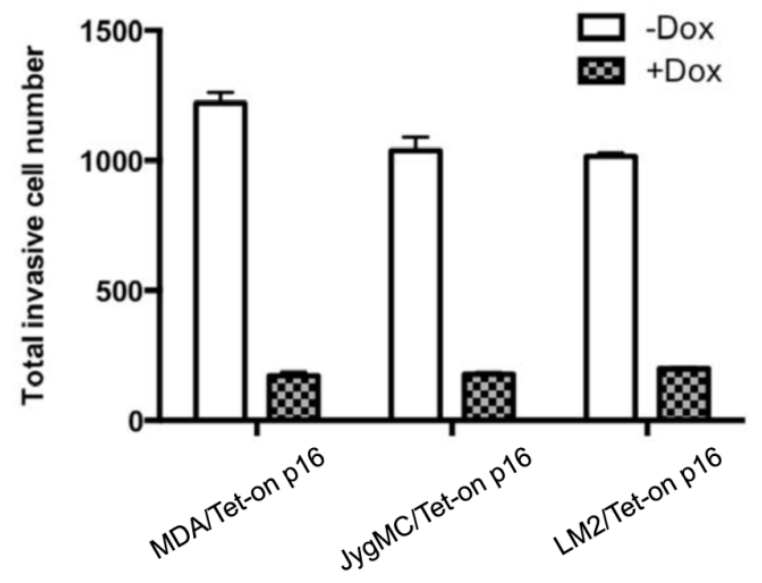

Figure 2. p16 inhibits breast cancer invasiveness. Various $B C$ a cells stably expressing Tet-on-p 16 were incubated with or without $1 \mu \mathrm{g} / \mathrm{ml} \mathrm{Dox} \mathrm{for}$ $72 \mathrm{~h}$, followed by invasion assay using commercial BD Biosciences Bio-Coat Matrigel Invasion Chamber according to the manufacturer's instructions. Results of duplicates are presented. Some error bars are too small to be shown at this scale.

\section{Effects of surrounding fibroblasts, and p16's neutralization, on cocultured $\mathrm{BCa}$ cell inva- siveness}

Emerging evidence implies that tumor-surrounding areas contribute to tumor growth and progression; however, the key attributes from tumor-surrounding cells that clearly display promotion for tumor progression as well as the relevant mechanisms of action remain unknown. To investigate effects of tumor-surrounding cells such as fibroblasts on BCa progression and invasion, we used the in vitro invasion assay to analyze effects of MEF fibroblasts on cocultured BCa LM2 cells' ability to penetrate the matrigel. After 18 hours incubation at $37^{\circ} \mathrm{C}$ of the LM2 alone or cocultured LM2 and MEF on the upper chamber of the insert that were precoated with matrigel (see details of $\mathrm{M} \& \mathrm{M}$ section), the matrigel (containing the noninvasive cells) was carefully removed from the upper surface of the membrane, the invaded cells (i.e., the matrigel-penetrated cells) remaining on the membrane were counted by a fluorescence microplate reader. Because LM2 cells express a GFP reporter gene (15), the fluorescence intensity (RFU) readout on the penetrated cells would be reflected only from BCa cells, not MEFs. As shown in Fig. 3, by comparing to LM2 cells alone, MEF-cocultured LM2 cells had a 4.3-fold increased invasiveness, demonstrating that surrounding fibroblasts stimulated cocultured BCa cell invasion.

To examine whether induced p16 could also inhibit the MEF-stimulated invasiveness, LM2/Tet-on-p16 with or without Dox induction were used for a similar assay. Consistently, p16 induction in the cocultured LM2 cells also significantly decreased invasiveness of the cocultured BCa cells (Fig.
4A). To exclude the possibility that Dox incubation per se might alter the BCa cell invasiveness, we also used parental LM2 cells cocultured with MEF for the same assay; we did not observe any effect from Dox treatment alone on the change of LM2 cell invasiveness (Fig. 4B).

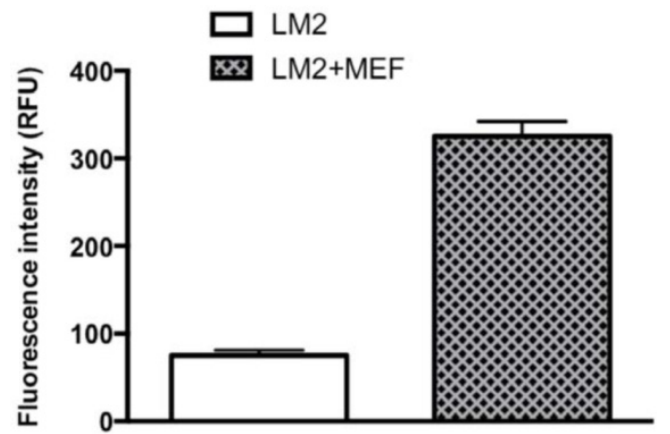

Figure 3. Cocultured MEFs stimulate LM2 cells' invasive ability. The BCa LM2 cells incubated with or without fibroblasts MEF were analyzed to measure the LM2 ability to penetrate matrigel (invasiveness) by counting the fluorescence (RFU). LM2 cells express a GFP gene. The invasion assay was performed under normoxia as described in $M$ \& $M$ section. The results represent the data from at least two independent experiments, each performed in duplicate.

$4 \mathrm{~A}$

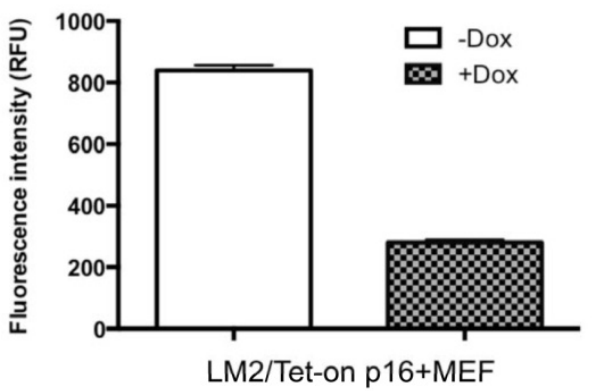

4B

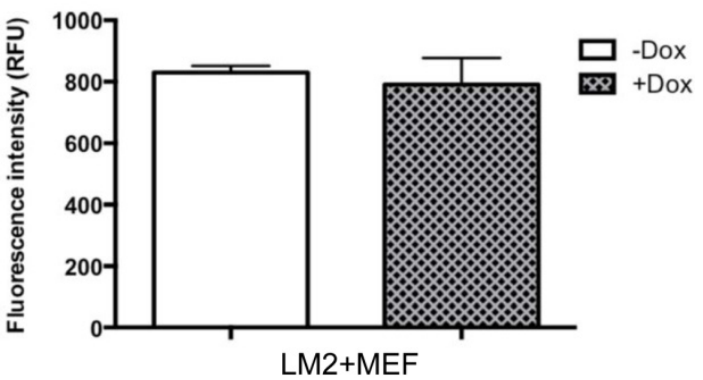

Figure 4. p1 6 inhibits MEF-stimulated invasiveness of LM2 cells under hypoxia. (4A), LM2/Tet-on-p16 cells were grown in culture medium with or without $1 \mu \mathrm{g} / \mathrm{ml}$ Dox for $72 \mathrm{~h}$, followed by cocultured with MEF. The LM2 cells' invasive ability was measured in terms of penetrating matrigel (invasiveness) by counting the fluorescence (RFU). (4B), Dox per se does not affect invasive ability of LM2 cells. 
Hypoxia and cocultured fibroblasts have additive effects on stimulation of BCa cell invasion ability

To examine whether hypoxia stimulates BCa cell invasiveness, we analyzed the matrigel-penetrating ability of LM2 cells in the presence or absence of cocultured MEF, under hypoxia or normoxia conditions. We found that hypoxia stimulated LM2 invasive ability at either LM2 growing alone (2.7-fold increase, compare column 1 and 2, Fig. 5) or coculturing with MEF (1.3-fold increase, compare column 3 and 4, Fig. 5). Consistent with what we observed earlier, MEF significantly simulated BCa cell invasion ability, either at normoxic condition (4.4-fold increase, compare column 1 and 3, Fig. 5) or hypoxia (2.2-fold increase, compare column 2 and 4, Fig. 5). The combination of hypoxia and cocultured MEF had apparent additive effects on stimulation of BCa LM2 invasive ability (5.9-fold increase, compare column 1 and 4).

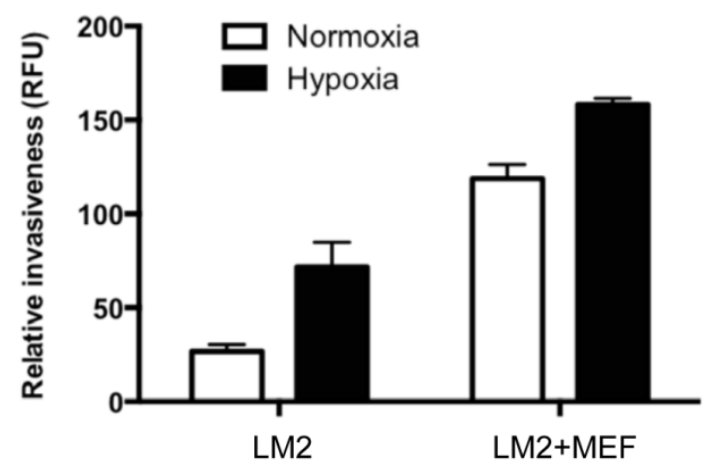

Figure 5. Hypoxia and cocultured MEF have additive effects on stimulation of LM2 cell invasion. The breast cancer LM2 cells cocultured with or without fibroblasts MEF and incubated under normoxia or hypoxia were analyzed to measure the LM2 ability to penetrate matrigel (invasiveness) by counting the fluorescence (RFU). The results (relative invasiveness) represent the data from at least two independent experiments, each performed in duplicate.

\section{HIF-1 a from fibroblasts contributes to $\mathrm{BCa}$ cell invasion in coculture}

Tumor-surrounding stromal cells (such as fibroblasts) are integral components of the tumor environment that together as an entity plays critical roles in the tumor progression. As both hypoxia and cocultured fibroblasts MEF stimulated BCa LM2 invasiveness (see above), we further investigated whether HIF-1a from tumor-surrounding fibroblasts cells plays a critical role for BCa cell invasion. We used a similar coculture setting as described above, with both HIF-1a WT MEF and HIF-1a KO MEF lines, respectively, to coculture with LM2 cells for the invasion assay. We found that HIF-1a WT MEF had a higher stimulation on cocultured LM2 invasiveness than its HIF-1a KO counterpart did (Fig. 6); by com- pared with LM2 alone, HIF-1a WT MEF had a stimulation of 10.9-fold increase of LM2 invasiveness, whereas HIF-1a KO MEF had 7.1-fold increase. These results implied that at least part (the difference of about 3.8-fold, Fig. 6) of the stimulation of LM2 invasion from the cocultured MEF was from HIF-1a effect of the surrounding MEF cells.

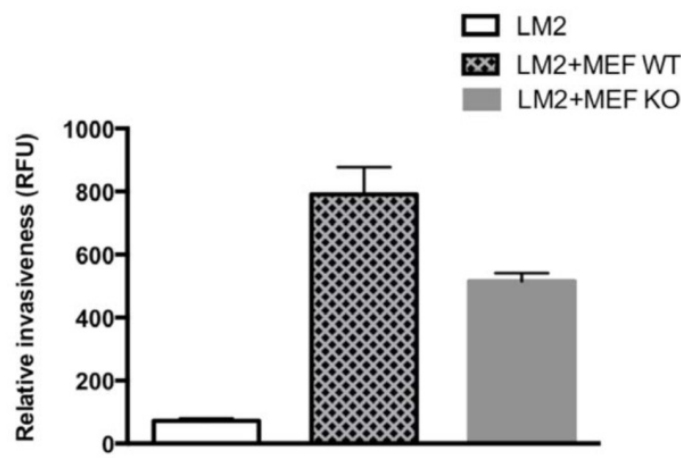

Figure 6. The effect of HIF-1 $\alpha$ expression from cocultured MEFs on BCa LM2 cells' invasiveness. The BCa LM2 cells cocultured with HIF-1 $\alpha$ WT MEFs or HIF-1 $\alpha$ KO MEFs were analyzed to measure the LM2 ability to penetrate matrigel by counting the fluorescence (RFU). The invasion assay was performed under normoxia as described in $M$ \& $M$ section. The results (relative invasiveness) represent the data from at least two independent experiments, each performed in duplicate.

\section{Discussion}

Despite the fact that $\mathrm{p} 16$ has been studied extensively on its anti-tumor cell proliferation properties and related function as a negative cell-cycle regulator, the link between p16 and hypoxia-stimulated tumor progression remains unclear. Specifically, exploration of p16's effects on cancer cell migration and invasion has barely begun. In this report, we investigated novel properties of $\mathrm{p} 16$ 's functions on its anti-migration and anti-invasion abilities of BCa cells. We found that p16 is capable of inhibiting hypoxia-induced cell migration and invasion of $\mathrm{BCa}$ cells, and suppressing cocultured fibroblast-stimulated invasiveness of $\mathrm{BCa}$ cells.

We have previously reported that p16 downregulates VEGF gene expression and $\mathrm{BCa}$ angiogenesis by neutralizing HIF-1a transactivation on VEGF pathway $(12,14)$. Consistent with our finding, a recent publication (18) revealed that p16 appears to repress the migration/invasion abilities of breast stromal fibroblasts and their VEGF-dependent angiogenesis through inhibition of Akt, a component of PI3K/Akt signaling pathway that regulates HIF-1a $(19,20)$. Moreover, silencing HIF-1a in malignant gliomas effectively inhibited cell migration and invasion under hypoxic environment, suggesting that HIF-1a expression induced by hypoxia is an essential event in the activation of glioma cell motility and invasion (21). 
Furthermore, a recently identified HIF-1a-direct target gene, HEF1 (human enhancer of filamentation 1), was shown to mediate hypoxia-induced migration of colorectal cancer cells (22). HEF1 was originally found as a scaffolding protein but recent data implicated it also acts as a key player in cancer cell migration and invasion (22-25). Elevated levels of HEF1 were found in various human cancers including BCa (26) and its expression proportionally correlates to tumor grade (27) and metastasis ability (28). Our unpublished results revealed that p16 inhibited hypoxia/HIF-1a stimulated HEF1 promoter transactivation (not shown). One possibility is that p16 may block the axis of "Hypoxia-HIF-1a-HEF1-migration/invasion" by neutralizing HIF-1a's transactivation on HEF1 gene. We are in the process to further elucidate the mechanism of action of p16-mediated inhibition on BCa cell migration and invasion.

Although several studies suggested that tumor-surrounding microenvironment contribute to tumor growth and progression, the key attributes from tumor-surrounding cells that clearly display promotion for tumor progression have yet to be specified. While many studies have reported that HIF-1 $\alpha$ protein in cancer cells plays an important role in cancer progression and various aspects of the tumor metastatic cascade, no study has yet reported on whether HIF-1 $\alpha$ expression from tumor-surrounding cells contributes to tumor progression. We demonstrate here, for the first time, that HIF-1 $\alpha$ from cocultured fibroblasts plays a critical role in promoting $\mathrm{BCa}$ cell invasion (Fig. 6). Consistent with our finding that HIF-1 $\alpha$ from tumor-surrounding fibroblasts plays a critical role in promoting tumor cell invasion (Fig. 6), our recent data, in a 3-D culture system containing BCa 4T1 cells cocultured with HIF-1 $\alpha$ WT or KO fibroblasts, revealed that HIF-1 $\alpha$ from fibroblasts plays a critical role under hypoxia for the 3-D BCa cells' protrusion formation (not shown). Protrusion formation is an established marker for invasive, aggressively malignant tumor type that start to invade the surrounding matrix in 3-D culture $(29,30)$. The increased invasiveness of BCa may be partially due to the increased secreted growth factors (such as VEGF that is upregulated by HIF-1 $\alpha$ ) in the tumor microenvironment from surrounding HIF-1 $\alpha-W$ T fibroblasts.

The development of intratumoral hypoxia is a common characteristic for fast-growing solid tumors including breast cancer. The intratumoral hypoxia activates HIF-1a in the tumor cells and its surrounding stromal cells, which in turn stimulate a group of HIF-1a-downstream genes responsible for tumor malignant progression including migration and invasion. Current chemotherapy for $\mathrm{BCa}$ treatment, including targeting the HIF-1a pathway, is not ideal due to toxic side effects of chemoagents and cancer resistance to them. One of the more promising approaches is the development of biologically based therapies to thwart the progression of metastatic disease (31). Specifically, a biological therapeutic approach that targets hypoxia-induced malignancy and directly neutralizes HIF-1a activity and its downstream genes mediating oncogenic pathways greatly deserves investigation. This study validates p16 as an effective biological agent to suppress hypoxia-promoted migration and invasion in BCa cells. The results of this study suggest that elevation and restoration of p16 in cancer microenvironment, either by gene therapy or pharmacology approaches, should provide a therapeutic mean to efficiently suppress BCa malignant progression.

\section{Abbreviations}

Breast cancer, BCa; Doxycycline, Dox; green fluorescent protein, GFP; hypoxia-inducible factor-1alpha, HIF-1a; immunohistochemistry, IHC; knockout, $\mathrm{KO}$; tetracycline-induced regulated promoter, Tet-on promoter; vascular endothelial growth factor, VEGF; wild-type, WT.

\section{Acknowledgments}

This research project was supported in part by NIH grant (CA107162) (YL) and University of Tennessee Health Science Center Bridge Funding.

\section{Competing Interests}

The authors have declared that no competing interest exists.

\section{References}

1. Friedl P, Wolf K. Tumour-cell invasion and migration: diversity and escape mechanisms. Nat Rev Cancer 2003; 3: 362-74.

2. Yamazaki D, Kurisu S, Takenawa T. Regulation of cancer cell motility through actin reorganization. Cancer Sci 2005; 96: 379-86.

3. Forsythe JA, Jiang $\mathrm{BH}$, Iyer NV, et al. Activation of vascular endothelial growth factor gene transcription by hypoxia-inducible factor 1. Mol Cell Biol 1996; 16: 4604-13.

4. Roth U, Curth K, Unterman TG, Kietzmann T. The transcription factors HIF-1 and HNF-4 and the coactivator p300 are involved in insulin-regulated glucokinase gene expression via the phosphatidylinositol 3-kinase/protein kinase B pathway. J Biol Chem 2004; 279: 2623-31.

5. Iliopoulos O, Levy AP, Jiang C, et al. Negative regulation of hypoxia-inducible genes by the von Hippel-Lindau protein. Proc Natl Acad Sci USA 1996; 93: 10595-9.

6. Semenza GL. Targeting HIF-1 for cancer therapy. Nat Rev Cancer 3: 721-732, 2003.

7. Chan DA, and Giaccia AJ. Hypoxia, gene expression, and metastasis. Cancer Metastasis Rev 2007; 26: 333-9.

8. Dvorak HF, Nagy JA, Feng D, et al. Vascular permeability factor/vascular endothelial growth factor and the significance of microvascular hyperpermeability in angiogenesis. Curr Top Microbiol Immunol 1999; 237: 97-132.

9. Hirota K, and Semenza GL. Regulation of hypoxia-inducible factor 1 by prolyl and asparaginyl hydroxylases. Biochem Biophys Res Commun 2005; 338: 610-6.

10. Wang GL, Jiang BH, Rue EA, and Semenza GL. Hypoxia-inducible factor 1 is a basic-helix-loop-helix-PAS heterodimer regulated by cellular $\mathrm{O} 2$ tension. Proc Natl Acad Sci USA 1995; 92: 5510-4.

11. Shapiro GI, Rollins BJ. p16INK4A as a human tumor suppressor. Biochem. Biophys. Acta 1996; 1242: 165-9.

12. Zhang J, Lu A, Li L, Yue J, Lu Y. p16 modulates VEGF expression via its interaction with HIF-1a in breast cancer cells. Cancer Invest 2010; 28: 588-97. 
13. Azuma H, Takahara S, Ichimaru N, et al. Marked prevention of tumor growth and metastasis by a novel immunosuppressive agent, FTY720, in mouse breast cancer models. Cancer Res 2002; 62: 1410-9.

14. Zhang J, Lu A, Beech D, Jiang B, Lu Y. Suppression of breast cancer metastasis through the inhibition of VEGF-mediated tumor angiogenesis. Cancer Ther 2007; 5: 273-86.

15. Kim M, Oskarsson T, Acharyya S, Nguyen DX, Zhang XH, Norton L, Massagué J. Tumor self-seeding by circulating cancer cells. Cell 2009; 139: 1315-26.

16. Choi HJ, Eun JS, Kim BG, et al. Vitexin, an HIF-1alpha inhibitor, has anti-metastatic potential in PC12 cells. Mol Cells 2006; 22: 291-9.

17. Wang Y, Li Z, Zhang H, et al. HIF-1alpha and HIF-2alpha correlate with migration and invasion in gastric cancer. Cancer Biol Ther 2010;10: 376-82.

18. Al-Ansari MM, Hendrayani SF, Tulbah A, Al-Tweigeri T, Shehata AI, Aboussekhra A. p16INK4A represses breast stromal fibroblasts migration/invasion and their VEGF-A-dependent promotion of angiogenesis through Akt inhibition. Neoplasia 2012; 14: 1269-77.

19. Jiang BH, Jiang G, Zheng JZ, Lu Z, Hunter T, Vogt PK. Phosphatidylinositol 3-kinase signaling controls levels of hypoxia-inducible factor 1. Cell Growth Differ 2001; 12: 363-9.

20. Zhou J, Schmid T, Frank R, Brune B. PI3K/Akt is required for heat shock proteins to protect hypoxia-inducible factor 1a from pVHL-independent degradation. J Biol Chem 2004; 279: 13506-13.

21. Fujiwara S, Nakagawa K, Harada H, Nagato S, Furukawa K, Teraoka M, Seno T, Oka K, Iwata S, Ohnishi T. Silencing hypoxia-inducible factor-1alpha inhibits cell migration and invasion under hypoxic environment in malignant gliomas. Int J Oncol 2007; 30: 793-802.

22. Kim SH, Xia D, Kim SW, et al. Human enhancer of filamentation 1 is a mediator of hypoxia-inducible factor-1alpha-mediated migration in colorectal carcinoma cells. Cancer Res 2010; 70: 4054-63.

23. O'Neill GM, Seo S, Serebriiskii IG, et al. A new central scaffold for metastasis: parsing HEF1/Cas-L/NEDD9. Cancer Res 2007; 67: 8975-9.

24. Li Y, Bavarva JH, Wang Z, et al. HEF1, a novel target of Wnt signaling, promotes colonic cell migration and cancer progression. Oncogene 2011; 30: 2633-43.

25. Bui L, Tomkiewicz C, Chevallier A, et al. Nedd9/Hef1/Cas-L mediates the effects of environmental pollutants on cell migration and plasticity. Oncogene 2009; 28: 3642-51.

26. Izumchenko E, Singh MK, Plotnikova OV, et al. NEDD9 promotes oncogenic signaling in mammary tumor development. Cancer Res 2009; 69: 7198-206.

27. Li Y, Bavarva JH, Wang Z, et al. HEF1, a novel target of Wnt signaling, promotes colonic cell migration and cancer progression. Oncogene 2011; 30: 2633-43.

28. Lucas JT, Salimath BP, Slomiany MG, Rosenzweig SA. Regulation of invasive behavior by vascular endothelial growth factor is HEF1-dependent. Oncogene 2010; 29: 4449-59.

29. Yamaguchi $\mathrm{H}$, and Condeelis J. Regulation of the actin cytoskeleton in cancer cell migration and invasion. Biochim Biophys Acta 2007; 1773: 642-52.

30. [Internet] Trevigen Cultrex 3D Spheroid Cell Invasion Assay (see the images of protrusions of MDA-MB-231 in Invasion Matrix. http://www.trevigen.com/item/8/51/0/761/Cultrex_3D_Spheroid_Cell_In vasion_Assay/

31. Steeg PS, Hartsough MT, and Clare SE. Nm23, breast differentiation, and cancer metastasis. In; Bowcock AM, ed. Breast Cancer. Totowa, New Jersey: Humana Press. 1998: 267-83. 